\title{
Zoonotic Sporotrichosis Epidemic Affects Children in Brazil
}

\author{
Cassio Porto Ferreira ${ }^{1}$ Ana Cristina Oliveira de Almeida ${ }^{2}$ Rodrigo de Almeida Paes ${ }^{3}$ Suzana Corte-Real ${ }^{4}$ \\ ${ }^{1}$ Department of Leprosy, Oswaldo Cruz Institute, Rio de Janeiro, Brazil \\ 2 Department of Structural Biology and Electron Microscopy, Oswaldo \\ Cruz Institute, Rio de Janeiro, Brazil \\ ${ }^{3}$ Department of Mycology, National Institute of Infectious Diseases, \\ Rio de Janeiro, Brazil \\ ${ }^{4}$ Department of Structural Biology and Electron Microscopy, Oswaldo \\ Cruz Institute, Rio de Janeiro, Brazil \\ J Pediatr Infect Dis 2015;10:25-26. \\ Address for correspondence Cassio Porto Ferreira, MD, MSc, Souza \\ Araújo Outpatient Clinic, Avenida Brazil, 4365-Manguinhos, Rio de \\ Janeiro 21045-900, Brazil (e-mail: drcassioferreira@yahoo.com.br).
}

\author{
Abstract \\ Keywords \\ - epidemic \\ - sporotrichosis \\ - zoonotic
}

Sporotrichosis is caused by the dimorphic fungus Sporothrix schenckii and was first described by Benjamin Schenck in 1898. It is the most common subcutaneous mycosis in Latin America. The infection is usually acquired by inoculation of the fungus through the skin. Zoonotic transmission of sporotrichosis seems to be rare worldwide. However, since 1998, an increasing number of cases have been reported in humans in Rio de Janeiro, Brazil; the vast majority of these cases have been associated with contact with cats affected by the same condition. Itraconazole is the drug of choice for treatment.

\section{Introduction}

Sporotrichosis, caused by the dimorphic fungus Sporothrix schenckii was first described by Benjamin Schenck in 1898. It is a subacute or chronic mycosis that affects all ages. ${ }^{1}$ It is currently distributed throughout the world, especially in tropical and subtropical zone, and is the most common subcutaneous mycosis in Latin America. Infection almost always occurs by traumatic inoculation of soil, plants, and organic matter contaminated by fungus. Felines have very close contact with contaminated soil and organic matter and constitute a reservoir of this agent. An epidemic of zoonotic transmission originating from infected domestic cats is occurring in Rio de Janeiro, Brazil. We present a case that exemplifies this problem.

\section{Case Report}

A 6-year-old boy presented with erythematous-crusted lesion on the trunk region, which had been present for 2 months ( - Fig. 1A, B). He gave a history of contact with a sick domestic cat (-Fig. 1C). An incisional biopsy of the lesion was performed, and histopathologic examination revealed pseudo-

received

July 20, 2014

accepted after revision

December 8, 2014

carcinomatous hyperplasia and occasional multinucleated giant cells. Growth of S. schenckii was obtained from culture of the tissue ( - Fig. 2A-D). After confirmation of the diagnosis of sporotrichosis treatment with itraconazole $100 \mathrm{mg} / \mathrm{d}$ was commenced, with healing after 3 months. The patient was regularly followed up after cessation of treatment, and no signs of recurrence were elicited.

\section{Discussion}

The outbreak of cat-related sporotrichosis in Rio de Janeiro, Brazil, appears to have begun in around 1998. Although several cases have been reported in the literature, ${ }^{1,2}$ the problem is not widely known about internationally. The rise in tourism in Brazil, particularly in association with major international sporting events, makes it important that health care practitioners in other countries are aware of this outbreak.

The presentation of sporotrichosis varies according to the virulence of the strain and host immune status. ${ }^{3}$ There is little direct evidence how the infectious agent became disseminated throughout the Rio de Janeiro municipality and its

Copyright $\odot 2015$ by Georg Thieme Verlag KG, Stuttgart · New York
DOI http://dx.doi.org/ 10.1055/s-0035-1554967. ISSN $1305-7707$. 

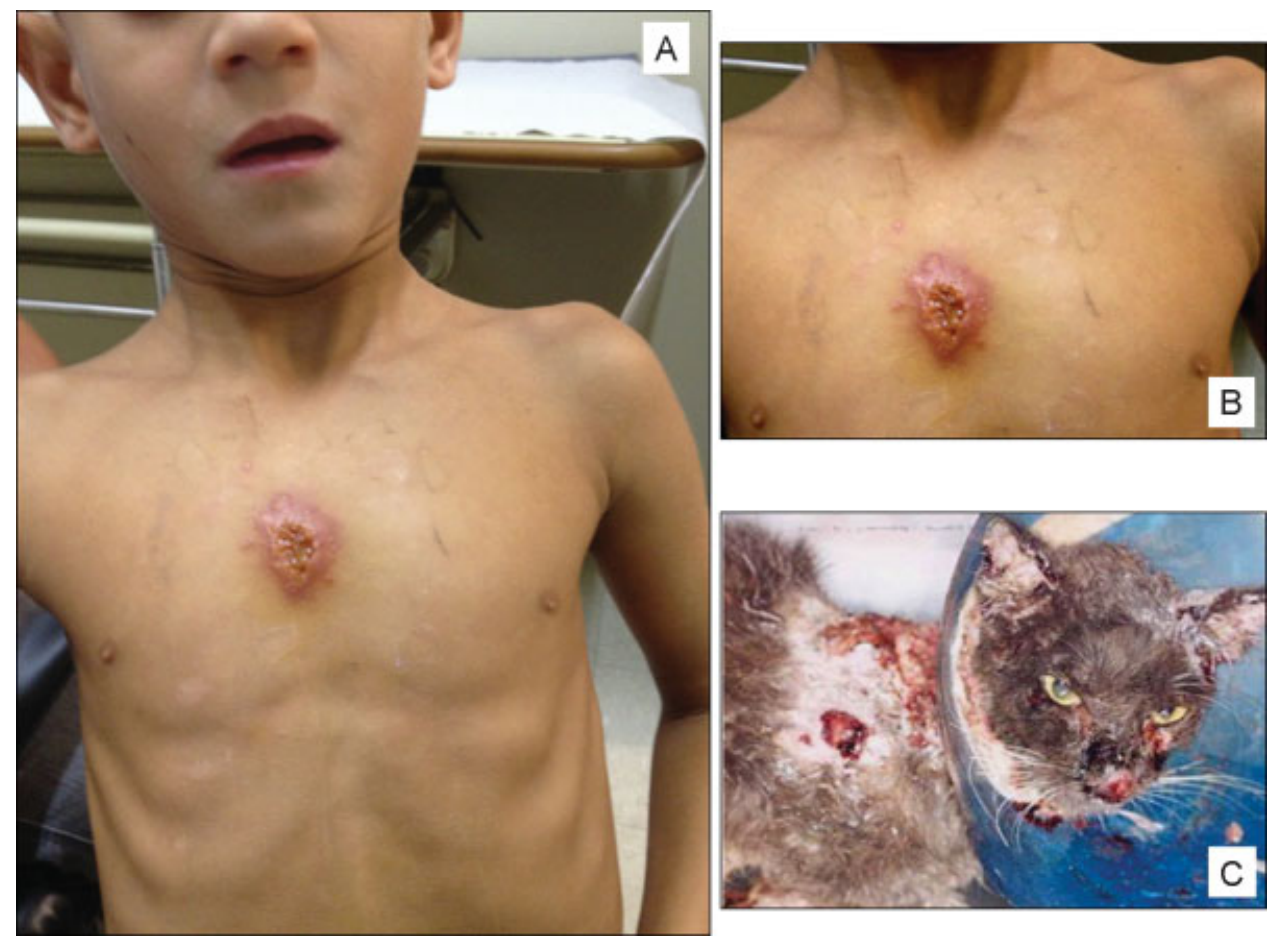

Fig. 1 (A) Erythematous crusted lesion on the trunk. (B) The same lesion in more detail. (C) An affected domestic cat from the sporotrichosis epidemic in Rio de Janeiro, Brazil.
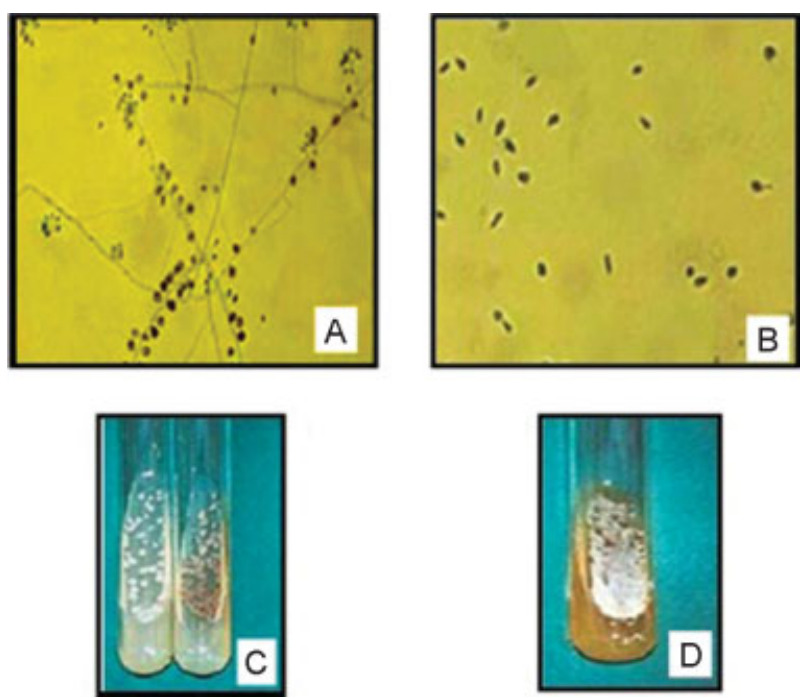

Fig. 2 The dimorphic fungus Sporothrix schenckii, growing: (A and C) as a mycelium at room temperature $\left(25^{\circ} \mathrm{C}\right)$, and $(B$ and $D)$ in the yeast phase $\left(37^{\circ} \mathrm{C}\right)$.

outskirts, but it is beyond reasonable doubt that the close interaction with cats represents a key route of transmission of the fungus to humans. ${ }^{1,2}$ However, felines have very close contact with contaminated soil and organic matter that constitute reservoirs of S. schenckii. ${ }^{4}$ The gold standard for sporotrichosis diagnosis is culture, and the drug of choice for treatment is oral itraconazole. ${ }^{5}$ Dermatologists and pediatrics must be aware of the possibility of cat-related sporotrichosis presenting in humans to avoid diagnostic pitfalls.

\section{Conflict of Interest \\ None.}

Role of Funding Source

None.

\section{References}

1 Schubach A, Barros MB, Wanke B. Epidemic sporotrichosis. Curr Opin Infect Dis 2008;21(2):129-133

2 Schubach AO, Schubach TM, Barros MB. Epidemic cat-transmitted sporotrichosis. N Engl J Med 2005;353(11):1185-1186

3 Mahajan VK, Sharma NL, Shanker V, Gupta P, Mardi K. Cutaneous sporotrichosis: unusual clinical presentations. Indian J Dermatol Venereol Leprol 2010;76(3):276-280

4 Dunstan RW, Reimann KA, Langham RF. Feline sporotrichosis. J Am Vet Med Assoc 1986;189(8):880-883

5 Lopes-Bezerra LM, Schubach A, Costa RO. Sporothrix schenckii and sporotrichosis. An Acad Bras Cienc 2006;78(2):293-308 